known disease are hypothesis-driven, where many scientists have contributed a great deal in terms of thought and experimental data. It is important not to let one scientist's undoubtedly questionable data blind us in our attempts to understand more about glaucoma and so realise more appropriate therapies. If the trend is to encourage the argument that a role for glutamate in glaucoma is fictional, then it may not be to the benefit of future glaucoma patients.

NN Osborne, G Chidlow and JPM Wood

Nuffield Laboratory of Ophthalmology, Oxford University, Oxford, UK

Correspondence: N Osborne,

Tel: + 441865 248996;

E-mail: Neville.Osborne@eye.ox.ac.uk

Eye (2006) 20, 1392-1394. doi:10.1038/sj.eye.6702234; published online 27 January 2006

Sir,

\section{Charles Bonnet syndrome (visual hallucinations) following enucleation}

We read with interest the excellent article by Drs Ross and Rahman, describing visual hallucinations in a patient following enucleation. ${ }^{1}$ These hallucinations were characteristic of Charles Bonnet syndrome (CBS) and, interestingly, disappeared with eye closure. We would like to propose a possible pathophysiologic mechanism to explain this observation.

A visual acuity of $6 / 6$ in the patient's other eye is not incompatible with the diagnosis of CBS, which has been described both in people with good visual acuity in the fellow eye $e^{2,3}$ and in patients who have visual field defects and good central visual acuity in the affected eye., ${ }^{2,5}$

Shiraishi et $a l^{6}$ proposed that it is the dynamic reduction in visual acuity, rather than the actual visual acuity, that has a greater impact on CBS., ${ }^{2,7}$

This case is intriguing because the hallucinations ceased when the patient's eyes were closed, only to return when he opened his eyes. Although it is well known that eye closure may terminate hallucinations in patients with CBS, this is the first case in which it appears that transient reduction of light perception on closure of the fellow eye is associated with its cessation. The enucleated eye constantly has no light perception and lid closure will not have any additional effect. It is possible, as the authors suggest, that closing the eyes results in secondary normalization of sensory input, thus abolishing the abnormal independent impulses and resultant complex imagery. ${ }^{1}$ Another possibility might be that deafferentation induced changes in the cortical neurons, resulting in reorganization of the receptive field and increased sensitivity to sensory input. ${ }^{8}$ Stimulation of these hypersensitive areas by normal sensory impulses (in this case from the left eye) may trigger visual hallucinations. ${ }^{7,9,10}$ However, a minimum amount of sensory input is required in order to trigger the hallucinations. Therefore, when the patient closes both his eyes, normal input is abolished and the hallucinations cease, only to return when he opens his eyes. This theory would also explain why some hallucinations cease when patients eventually lose all light perception. This possibility is illustrated in another patient who experienced CBS following cortical resection for cortical dysplasia. ${ }^{5}$ In this patient, the hallucinations diminished with eye closure, and varied in intensity with blinking, light intensity, and the sight of moving objects - factors that vary the intensity of the visual stimulation.

Regardless of the mechanism, we agree with the authors that it is important to recognize CBS and its possible occurrence following sudden loss of vision.

\section{References}

1 Ross J, Rahman I. Charles Bonnet Syndrome following enucleation. Eye 2005; 19(7): 811-812.

2 Tan CS, Lim VS, Ho DY, Yeo E, Ng BY, Au Eong KG. Charles Bonnet syndrome in Asian patients in a tertiary ophthalmic centre. Br J Ophthalmol 2004; 88: 1325-1329.

3 Holroyd S, Rabins PV, Finkelstein D, Lavrisha M. Visual hallucinations in patients from an ophthalmology clinic and medical clinic population. J Nerv Ment Dis 1994; 182: 273-276.

4 Freiman TM, Surges R, Vougioukas VI, Hubbe U, Talazko J, Zentner J et al. Complex visual hallucinations (Charles Bonnet syndrome) in visual field defects following cerebral surgery. Report of four cases. J Neurosurg 2004; 101(5): 846-853.

5 Choi EJ, Lee JK, Kang JK, Lee SA. Complex visual hallucinations after occipital cortical resection in a patient with epilepsy due to cortical dysplasia. Arch Neurol 2005; 62(3): 481-484.

6 Shiraishi Y, Terao T, Ibi K, Nakamura J, Tawara A. Charles Bonnet syndrome and visual acuity - the involvement of dynamic or acute sensory deprivation. Eur Arch Psychiatr Clin Neurosci 2004; 254(6): 362-364.

7 Tan CS, Sabel BA. Dynamic changes in visual acuity as the pathophysiologic mechanism in Charles Bonnet syndrome (visual hallucinations). Eur Arch Psychiatr Clin Neurosci 2005 August 4 (E-pub ahead of print).

8 Eysel UT, Schweigart G, Mittmann T, Eyding D, Qu Y, Vandesande $\mathrm{F}$ et al. Reorganization in the visual cortex after retinal and cortical damage. Restor Neurol Neurosci 1999; 15(2-3): 153-164.

9 Tan CS, Sabel BA. Charles Bonnet syndrome in patients with visual field defects - are hallucinations good or bad? J Neurosurg 2005; 103(1): 193-195 (author reply 195).

10 Tan CS, Sabel BA. Charles Bonnet syndrome after occipital cortical resection for cortical dysplasia may be related to denervation supersensitivity. Arch Neurol 2005; 62(9): 1479-1480. 
CSH Tan' ${ }^{1}$, BA Sabel ${ }^{2}$ and K-G Au Eong Ka, $^{1,3,5}$

${ }^{1}$ The Eye Institute, Tan Tock Seng Hospital, National Healthcare Group,

Singapore

${ }^{2}$ Institute of Medical Psychology, University of Magdeburg Medical School, Magdeburg,

Germany

${ }^{3}$ The Eye Institute, Alexandra Hospital, National Healthcare Group, Singapore

${ }^{4}$ Singapore Eye Research Institute, Singapore

${ }^{5}$ Department of Ophthalmology, National University of Singapore, Singapore

Correspondence: CSH Tan, Department of Ophthalmology,

The Eye Institute,

Tan Tock Seng Hospital,

11 Jalan Tan Tock Seng,

Singapore 308433, Singapore

Tel: + 6563577726 ;

Fax: + 6563577718 .

E-mail: Colintan_eye@yahoo.com.sg

The authors have not received any financial support in the preparation of this article and do not have any financial or proprietary interests in the article

Eye (2006) 20, 1394-1395. doi:10.1038/sj.eye.6702236; published online 27 January 2006

Sir,

\section{Reply to Tan et al}

We welcome and thank Tan et $a l^{1}$ for their interest and observations regarding our previously published case report.

We are in agreement regarding Snellen acuity not being a diagnostic criteria for Charles-Bonnet Syndrome (CBS). Numerous reports have highlighted the presence of CBS-type hallucinations in individuals with good Snellen acuity with restricted visual fields. It appears, therefore, that the quality of visual function and its deviation from the previous norm for the individual are key elements in production of CBS hallucinations. It is for this reason that we agree with the observation by
Holroyd $e a^{2}$ that sudden and abrupt reduction in visual function may trigger CBS symptoms.

Tan et $a l^{1}$ comment on reduction of light perception in the fellow eye terminating CBS symptoms in our case. We agree that the proposed theory could account for resolution of CBS on eye closure. This theory, however, is similar to the other commonly perceived theories of deafferentation described by Bartlett ${ }^{3}$ and West. ${ }^{4}$ The visual sensory cortex, when deprived of normal afferent input may exhibit spontaneous independent activity with resultant conscious images. This hypothesis is supported by the observation that such hallucinations may be abolished by normal or excessive visual stimulation. ${ }^{3}$ The perceptual theory of West describes afferent input reduction below a threshold level (such as in disease of the eyes or visual pathway), resulting in the brain allowing previously registered subconscious perceptions to emerge into consciousness, resulting in a hallucinatory experience. ${ }^{4}$

Similarly, as the hallucinations of CBS have only been documented in acquired visual deprivation, it is possible that in-built higher centre mechanisms are in place so that eye closure may trigger intracerebral activity suggesting to the sensory visual cortex that visual stimulation has ended, rather than sensory input itself being detected by sensory mechanisms to be decreased. This may explain why darkness with the eyes open may still result in CBS but darkness as a result of eye closure may obliterate CBS hallucinations.

\section{References}

1 Ross J, Rahman I. Charles Bonnet syndrome following enucleation. Eye 2005; 19: 811-812.

2 Holroyd S, Rabins PV, Finkelstein D, Nicholson MC, Chase GA, Wisniewski SC et al. Visual hallucinations in patients with macular degeneration. Am J Psychiatry 1992; 149: 1701-1706.

3 Bartlett JEA. A case of organised visual hallucinations in an old man with cataract, and their relationship to the phenomena of the phantom limb. Brain 1951; 74: 363-373.

4 West LJ. A clinical and theoretical overview of hallucinatory phenomena. In: Siegal RK, West LJ (eds). Hallucinations: Behaviour, Experience and Theory. John Wiley and Sons: New York, 1975, pp 287-311.

I Rahman

Ophthalmology, Manchester Royal Eye hospital, Lister Centre, Nelson Street, Manchester M13 9WL, UK

Correspondence: I Rahman, Ophthalmology, Manchester Royal Eye hospital, Lister Centre, Nelson Street, Manchester M13 9WL, UK 